\title{
ESTUDO ESPECTRO ELETROQUÍMICO DE SISTEMAS ESTRUTURADOS ARGILA-CORANTE
}

\author{
G. R. de SOUZA' ; F. L. FERTONANI'; I. A. PASTRE2.
}

\section{Resumo}

O comportamento eletroquímico e espectroeletroquímico do sistema montmorilonita-azul de

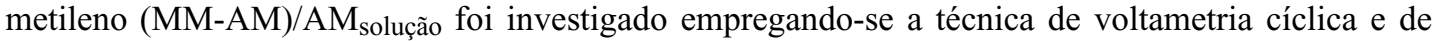
transiente, I x t (corrente versus tempo) sobre eletrodo de vidro ITO modificado pela deposição mecânica de filmes do compósito MM-AM. Os filmes foram preparados empregando três procedimentos: 1- na ausência de $\mathrm{CTACl}$ (surfactante, cloreto de cetiltrimetilamônio); 2- com incorporação do CTACl à região interlamelar; e 3- na presença do $\mathrm{CTACl}$, porém, não incorporado à região interlamelar. A técnica espectrofotométrica foi empregada para a caracterização e para o estudo eletrocrômico dos filmes formados. Os resultados experimentais permitiram observar que: i- os filmes preparados com a incorporação prévia do AM a região interlamelar fornecem uma resposta eletroquímica mais eficiente com o aparecimento de um par de picos bem definido; ii- a adição do CTACl, de forma geral permite a obtenção de filmes mecanicamente mais estáveis; iii- com a inclusão prévia do CTACl na região interlamelar não ocorre o aparecimento da forma protonada do $\mathrm{AM}\left(\mathrm{AMH}^{+}\right)$; iv- na ausência da forma protonada o compósito não apresenta comportamento eletrocrômico.

Palavras-chave: Argila, espectroeletroquímica, voltametria cíclica, montmorilonita, azul de metileno, filmes, eletrodos modificados.

\section{Introdução}

Argilas são minerais de ocorrência natural no solo e apresentam características físico-químicas peculiares. São formadas por pequenos cristais, quase sempre na forma de plaquetas hexagonais, que se aglutinam para formar conglomerados.

Essas plaquetas são constituídas de aluminossilicatos organizados, compostos pelos elementos de $\mathrm{Si}, \mathrm{Al}$ e oxigênio, além de outros em menores proporções, como o $\mathrm{Mg}$, $\mathrm{Fe}$ e $\mathrm{Ca}$, com formula geral: $(\mathrm{OH})_{4} \mathrm{Si}_{8}\left(\mathrm{Al}_{3,34} \mathrm{Mg}_{0,66}\right) \mathrm{O}_{20}$.
Quando em suspensão, em água, permite a separação de suas camadas lamelares e a intercalação de íons metálicos (metais pesados) e moléculas (solvente, moléculas orgânicas, etc.) [8]. $\mathrm{O}$ entendimento das propriedades de sistemas organizados, tais como: a formação de sistemas micelares, a presença de polieletrólitos interagindo com as argilas, a formação de sistemas vesiculares, e a grande aplicação industrial das argilas leva ao interesse pelo estudo de sistemas estruturados constituídos pelas argilas.

Sistemas estruturados do tipo

\footnotetext{
${ }^{1}$ Depto. Quím. Analítica - Instituto de Química-UNESP- CEP 14801-440 - Araraquara - SP - Brasil

${ }^{2}$ Depto. Quím. e Geociências - IBILCE-UNESP - CEP 15054-000 - S. J. do Rio Preto - SP - Brasil
} 
argilomineral-pesticidadas, empregando os compostos metilviologen, paraquat, etc., tem sido encontrados na literatura como modelo para o entendimento das interações dos pesticidas com argilominerais $[16,17,20]$. Recentemente, sistemas do tipo herbicida-compósito argilomineralhexadeciltrimetilamônio (CTAB) tem sido conduzidos com o objetivo de preparar sistemas que permitam controlar a disponibilidade dos herbicidas, alterando a solubilidade dos herbicidas em água, sua fotoestabilidade, etc., o que permitiria minimizar os efeitos de contaminação de águas superficiais e de subsolo $[5,6,15,18]$.

Eletrodos modificados com compósitos argilominerais vêm sendo desenvolvidos de longa data $[4,7,9,10-14,19,22,24-28]$, empregando distintos substratos $(\mathrm{Pt}$, carbono vítreo, eletrodo de pasta de carbono, etc.) e objetivando diferentes aplicações tais como: 1- reconhecimento óptico; 2desenvolvimento de sistemas eletrocatalíticos; 3desenvolvimento de eletrodos empregados para determinações eletroanalíticas.

Desta forma, considerando as aplicações historiadas anteriormente o presente trabalho tem como objetivo estudar o comportamento espectroeletroquímico de filmes montmorilonitaazul de metileno (MM-AM) gerados sobre eletrodos de vidro ITO.

\section{Procedimento Experimental}

\section{Equipamentos}

- Espectrofotômetro: HP 8453, diodo "array" interfaceado a um computador compatível com a linha IBM-PC e "software" para aquisição e tratamento de dados.

- Potenciostato: ECOCHIMIE PGSTAT10, interfaceado a um computador compatível com a linha IBM-PC, e "software" GPES3, ECOCHIMIE para aquisição e tratamento de dados.

- Célula eletroquímica: os eletrodos de trabalho, auxiliar e de referência foram montados no interior de uma cubeta de quartzo de $5 \mathrm{~mL}$. A tampa de teflon, da célula foi cortada e perfurada de modo a acomodar os eletrodos.

- $\quad$ Eletrodo de referência de $\mathrm{Ag} / \mathrm{AgCl} / \mathrm{NaCl}(0,050$ mol L-1), preparado em uma ponteira de micropipeta, para adaptação a célula. $O$ contato elétrico era obtido mantendo-se a extremidade inferior da ponteira aberta.

- Contra eletrodo: foi empregado um fio de Pt em forma de retângulo, acompanhando o perfil interno da cubeta e colocado de frente para o lado ativo do vidro ITO.

- Eletrodo de trabalho: eletrodo de vidro ITO recoberto com o filme de MM-AM.

\section{Procedimentos para a modificação dos eletrodos de vidro ITO}

I- Preparo do compósito montmorilonita - azul de metileno (MM-AM): agitou-se por $24 \mathrm{~h}$ uma suspensão metanólica de montmorilonita (MM), na presença do corante azul de metileno (AM, $1 \%$, m/ $\mathrm{m})$, eliminando-se o solvente em corrente de $\mathrm{N}_{2}$ até a secura.

II- Preparo das suspensões de montmorilonita (MM) e do compósito MM-AM: a serem aplicados sobre o eletrodo de vidro ITO, para a formação do filme: 1) suspensão aquosa de MM, 2 g L-1, agitada por 12 $\mathrm{h}$; 2) suspensão aquosa de MM-AM, $2 \mathrm{~g} \mathrm{~L}^{-1}$ (preparada em I), agitada por $12 \mathrm{~h} ; 3$ ) suspensão aquosa de MM-AM, $2 \mathrm{~g} \mathrm{~L}^{-1}$ (preparada em I), agitada por $12 \mathrm{~h}$ na presença do surfactante cloreto de cetil-trimetilamônio (CTACl) $(12 \mu \mathrm{L}: 3 \mathrm{~mL})$; 4) suspensão aquosa de MM $\left(2 \mathrm{~g} \mathrm{~L}^{-1}\right)$ tratada com CTACl $(12 \mu \mathrm{L}: 3 \mathrm{~mL}), \operatorname{AM}(1 \%, \mathrm{~m} / \mathrm{m})$ e agitada por 15 h. Posterior à agitação, as suspensões foram centrifugadas a $1000 \mathrm{rpm}$ por $5 \mathrm{~min}$ e o sobrenadante empregado para a geração dos filmes.

III- Modificação da superfície dos eletrodos: sobre o vidro ITO $\left(\mathrm{a}_{\mathrm{g}}=0,56 \mathrm{~cm}^{2}\right)$ aplicou-se incrementos de $10 \mu \mathrm{L}$ da suspensão, em circulo, de modo a cobrir toda a área superficial do eletrodo em uma única gota de $100 \mu \mathrm{L}$. Posteriormente, o solvente foi evaporado em estufa a $80^{\circ} \mathrm{C}$, por 40 minutos. Este procedimento foi procedimento repetido 3 vezes para o mesmo eletrodo, para evitar descontinuídade nos filmes.

IV- Estudo do comportamento eletroquímico do sistema MM-AM/AM(em solução aquosa): foram obtidos voltamogramas cíclicos (VC) para as

Ecl. Quím., São Paulo, 28(1): 77-83, 2003 
seguintes condições:

a) $\mathrm{E}_{\text {inic. }}=\mathrm{E}_{\text {fin. } .3}=0,4 \mathrm{~V} ; \mathrm{E}_{\text {inv. } 1}$ e $\mathrm{E}_{\text {inv. } 2}=$ variável;

b) $(0,05 \leq \mathrm{v} \leq 0,2) \mathrm{V} \mathrm{s}^{-1}$; em meio de $\mathrm{NaCl} 0,050 \mathrm{~mol}$ $\mathrm{L}^{-1}$, para $\left(0,8<\mathrm{C}_{\mathrm{AM}}<2,4\right) \times 10^{-5} \mathrm{~mol} \mathrm{~L}^{-1}$.

V- Estudo do comportamento espectral (UVvisível): foram obtidos espectros dos filmes preparados sobre o vidro ITO para estudar a presença das espécies protonadas e não protonadas e o seu efeito sobre os perfis dos voltamogramas cíclicos.

VI- Estudo do comportamento espectroeletro químico do filme de MM-AM em solução de eletrólito de suporte: o filme, após a deposição, foi imerso em solução de $\mathrm{NaCl} 0,050 \mathrm{~mol} \mathrm{~L}^{-1}$, desaerado com $\mathrm{N}_{2}$, por $5 \mathrm{~min}$, e submetido a transientes I x t, de duplo salto com simultânea obtenção do espectro UV-visível. O transiente de duplo salto foi obtido de acordo com o seguinte programa de potenciais: $\mathrm{E}_{1}=0,20 \mathrm{~V}, \mathrm{t}_{1}=\operatorname{variável}(60 \mathrm{a} 900 \mathrm{~s}) ; \mathrm{E}_{2}=$ variável $(0,10 \mathrm{a}-0,50 \mathrm{~V} ; \Delta \mathrm{E}=20 \mathrm{mV}), \mathrm{t}_{2}=\operatorname{variável}(60$ a $900 \mathrm{~s}$ ); os espectros foram obtidos ao final do tempo do transiente $\left(\mathrm{t}_{1}, \mathrm{t}_{2}-5\right) \mathrm{s}$.

\section{Resultados e discussões}

As Figuras 1a) e b) apresentam os VCs obtidos sobre o eletrodo de vidro ITO modificado empregando suspensão aquosa de montmorilonita, $\mathrm{MM}\left(2 \mathrm{~g} \mathrm{~L}^{-1}\right)$ (metodologia II-1), e suspensão aquosa do compósito MM-AM (preparado pela metodologia II-2), ambos na ausência do CTACl.

A Figura 1a) foi obtida em solução do eletrólito de suporte (curva 1) e em solução de AM (8 e $16 \mu \mathrm{mol} \mathrm{L}^{-1}$; curvas 2,3). Inicialmente, observase a mudança de coloração do filme (incolor $\rightarrow$ azul) devido a adsorsão do monômero, espécie AM, e o aparecimento gradual do par redox, representado por $\mathbf{A}(\mathrm{Ec}=-0,06 \mathrm{~V})$ e $\mathbf{B}(\mathrm{Ea}=-0,13 \mathrm{~V})$, com o aumento do número de ciclos (50 ciclos - curva 3; $\left.\mathrm{CAM}=16 \mu \mathrm{mol} \mathrm{L}^{-1}\right)$. A Figura 1b), curva 1, apresenta um pico catódico em $-0,07 \mathrm{~V}$, cuja intensidade aumenta nos 2 primeiros ciclos posterior à adição do $\mathrm{AM}$ ( curva 2; $\mathrm{CAM}=8 \mu \mathrm{mol}$ $\mathrm{L}^{-1}$ ). Com o aumento do número de ciclos verificase o aparecimento do par de picos A $(-0,21 \mathrm{~V}), \mathrm{B}(-$ $0,32 \mathrm{~V}$ ), e a conseqüente estabilização do $\mathrm{VC}$ após o $15^{\circ}$ ciclo (curva 3). É importante observar que a razão $\mathrm{ip}^{\mathrm{a}} / \mathrm{ip}^{\mathrm{c}} \sim 0,59$, sugerindo um processo de transferência de elétrons facilitada.
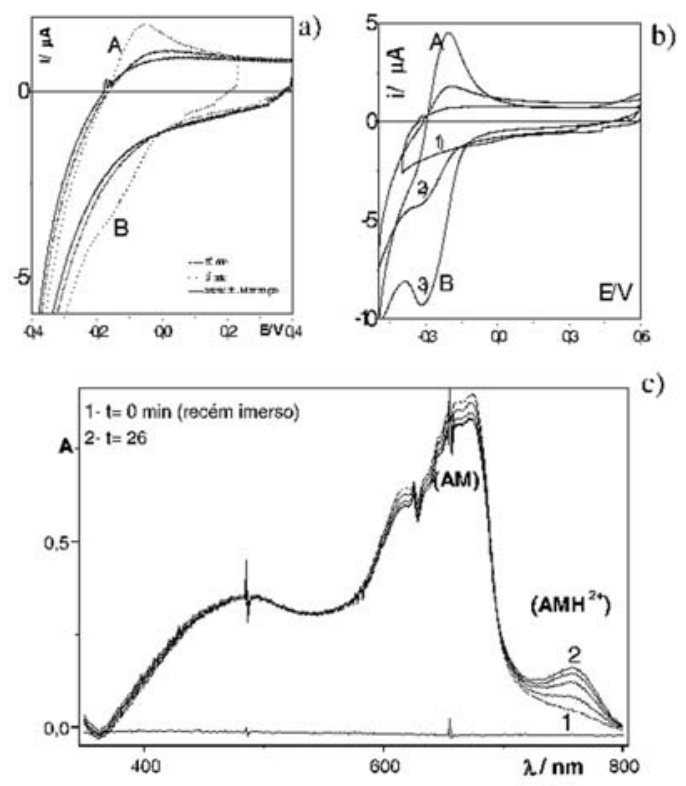

Figura 1: VCs obtidos para os eletrodos ITO modificados com: a) MM: (_) branco, (_.._) 8 e (......) $16 \mu \mathrm{mol} \mathrm{L}{ }^{-1}, \mathrm{AM}, 3^{\circ}$ e $50^{\circ}$ ciclo, respectivamente; b) compósito MM-AM: 1) branco, 2) $3^{\circ}$, 3) $15^{\circ}$ ciclo, 8 $\mu \mathrm{mol} \mathrm{L}{ }^{-1}, \mathrm{AM}$; c) espectro UV-visível obtido para o filme de $M M$, recém recoberto por $\mathrm{AM}$, para constatação do processo de difusão do AM para a região interlamelar com o tempo; eletrodo imerso em solução de $\mathrm{NaCl} \quad 0,050$ mol L ${ }^{-1}$; intervalo de tempo para obtenção dos espectros: $\Delta \mathrm{t}=1 \mathrm{~min} . \mathrm{T}=(25 \pm 1){ }^{\circ} \mathrm{C}$.

Na Figura 1c) estão representados os espectros obtidos para um filme de MM-AM preparado de modo a minimizar o "inchamento" das lamelas e dificultar a migração do monômero do azul de metileno (AM) para os sítios ácidos da argila. Os espectros foram obtidos em intervalos de tempo $\triangle \mathrm{t}=1 \mathrm{~min}$, posterior a imersão do eletrodo em solução de $\mathrm{NaCl} 0,050 \mathrm{mmol} \mathrm{L}^{-1}$, permitindo observar o aparecimento e a intensificação da banda de absorção do monômero protonado, espécie $\mathrm{AMH}^{2+}$ em $750 \mathrm{~nm}[1,3,21]$.

A Figura 2a) apresenta os VCs obtidos para o AM, $30 \mu \mathrm{mol} \mathrm{L}^{-1}$ em solução de $\mathrm{NaCl} 0,050$ mol L-1, para $(0,050 \leq \mathrm{v} \leq 0,20) \mathrm{V} \mathrm{s}^{-1}$. Os VCs foram obtidos sobre o eletrodo modificado empregandose a metodologia de depósito II-3 (surfactante adicionado à suspensão do compósito MM-AM).

A Figura 2, c1), apresenta o espectro de absorção obtido para o filme da Figura 2a), onde 
são observadas as bandas de absorção características das espécies: 1) AM em 660 nm;2) $\mathrm{AMH}^{2+}$ em $750 \mathrm{~nm}$ (resultado da migração do AM para os sítios ácidos da argila durante o processo de suspensão, metodologia I [1,3,21]). A presença destas espécies, nos diferentes sítios da argila, revela que o AM quando pré-adsorvido não pode ser deslocado pelo surfactante [2].
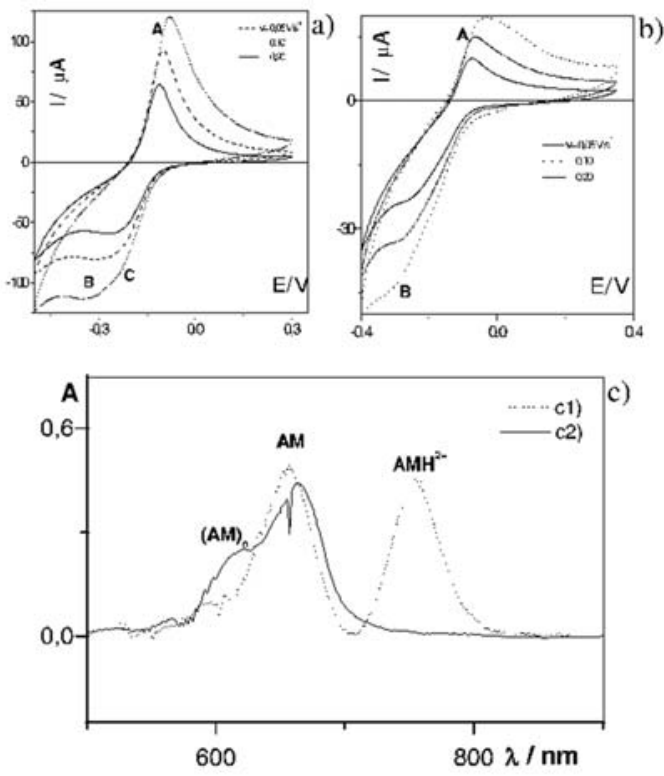

Figura 2. VCs do AM $30 \mu \mathrm{mol} \mathrm{L}^{-1}$ em solução de $\mathrm{NaCl}$ 0,050 mol L-1 obtido empregando eletrodo ITO modificado a partir das metodologias: a) II-3, ausência do $\mathrm{CTACl}$ na região interlamelar; b) II-4, presença do CTACl na região interlamelar. c) Espectros de absorção na região do visível obtidos para os filmes empregados em a) c1; b) c2. $\mathrm{T}=(25 \pm 1){ }^{\circ} \mathrm{C}$

A Figura 2b) apresenta os VCs obtidos para o filme preparado pela metodologia II-4, situação na qual a suspensão de argila é agitada simultaneamente na presença do surfactante e do AM $(1 \% \mathrm{~m} / \mathrm{m})$; condições experimentais idênticas às da Figura 2a). Na Figura 2, c2) está representado o espectro de absorção do referido filme, revelando a ausência do monômero protonado do corante $\left(\mathrm{AMH}^{2+}\right)$, como resultado da ocupação preferencial dos sítios ácidos da argila pelo CTACl [2]. A ausência da espécie $\mathrm{AMH}^{2+}$ está em concordância com os resultados apresentados na Figura 1c) considerando-se que com a ocupação dos sítios ácidos, não ocorre a difusão do AM para a região interlamelar da MM.

A comparação dos VCs apresentados nas Figuras 2a) e 2b) revela um perfil I x E ligeiramente semelhante, com um conjunto de picos redox (A, B). Porém, os VCs da Figura 2a) apresentam intensidades de corrente consideravelmente superiores e uma razão ipa / ipc $\sim$ 1,04. A Figura 2b), por sua vez, apresenta uma razão ip $\mathrm{ip}^{\mathrm{a}} \mathrm{ip}^{\mathrm{c}} \sim 0,53$, sugerindo um processo de transferência de elétrons facilitada, apesar das baixas correntes.

Os VCs da Figura 2a) apresentam um conjunto de picos superpostos na região catódica, não observados na Figura 2b). A desconvolução deste conjunto de picos permitiu a determinação dos respectivos valores de potencial $(\mathrm{E})$ de pico: $\mathrm{C}$ $\left(E=0,23 \mathrm{~V} ; \mathrm{i}_{\mathrm{p}} \mathrm{a}=42 \mu \mathrm{A}\right) ; \mathrm{B}\left(\mathrm{E}=0,37 \mathrm{~V} ; \mathrm{i}_{\mathrm{p}} \mathrm{a}=94 \mu \mathrm{A}\right) . \mathrm{A}$ correlação $\mathrm{i}_{\mathrm{p}}^{\mathrm{a}} \times \mathrm{v}^{1 / 2}$ foi observada para os picos de oxidação $(\mathrm{A} \sim 0,1 \mathrm{~V})$ e de redução $(\mathrm{B}, \mathrm{C})$, para ambos os sistemas, demonstrando tratar-se de um processo controlado por difusão. A correlação $\mathrm{i}_{\mathrm{p}}{ }^{\mathrm{a}}$ e $i_{\mathrm{p}} \mathrm{c} \times \mathrm{C}_{\mathrm{AM}}$ obtida para $\left(5 \leq \mathrm{C}_{\mathrm{AM} \text { solução }} \leq 30\right)$ $\mu \mathrm{mol} \mathrm{L}-1$ e $\mathrm{v}=0,1 \mathrm{~V} \mathrm{~s}^{-1}$, para os eletrodos preparados pelas metodologias II-3 e II-4, forneceu relações lineares, porém, o filme preparado pela metodologia II-4 apresentou desvio da linearidade para baixos valores de $(5 \leq \mathrm{CAM} \leq 10) \mu \mathrm{mol} \mathrm{L} \mathrm{L}^{-1}$.

As Figuras 3a) e b) apresentam os espectros de absorção para os eletrodos de filme de MM-AM modificados empregando as metodologias II-3 e II-4, na ausência e na presença do CTACl na região interlamelar, respectivamente. Os espectros foram obtidos para os eletrodos imersos em solução de $\mathrm{NaCl}, 0,050 \mathrm{mmol} \mathrm{L}^{-1}$, empregando diferentes programas de perturbação de potenciais $(\mathrm{I} \times \mathrm{t})$.

$\mathrm{Na}$ Figura 3a) estão representados os espectros de absorção obtido para o eletrodo de vidro ITO modificado com o filme do compósito MM-AM, contendo o CTACl na região interlamelar.

Os espectros foram obtidos para os transientes, Ix t, no intervalo de $\mathrm{E}_{2}=0,10 \mathrm{a}-0,50 \mathrm{~V}$, $\Delta \mathrm{E}=20 \mathrm{mV}$, para tempo de amostragem de corrente, $\mathrm{ta}=60 \mathrm{~s}$, com exceção da curva 1 (ausência de potencial aplicado (Eap.)) e da curva $10, \mathrm{E}_{2}=-0,4 \mathrm{~V}$ e tempo de amostragem de corrente, ta $=900 \mathrm{~s}$. A figura não revela a atenuação da intensidade dos picos das espécies absorventes: agregados (AM)n 
em 600 nm) e monômero (AM em 660 nm) com o avanço do $\mathrm{E}_{2}$ para a região catódica, como observado na literatura para os sistemas AM nafion e polianilina - AM - nafion $[10,12,13]$, situação para a qual todo o azul de metileno foi reduzido à forma incolor (forma leuco).

Assim, a ausência de atenuação dos picos de absorção revelada a partir dos resultados da Figura 3a) sugere que para este sistema não ocorre a geração eletroquímica da forma leuco do azul de metileno, estando presente apenas as formas não protonadas do AM.
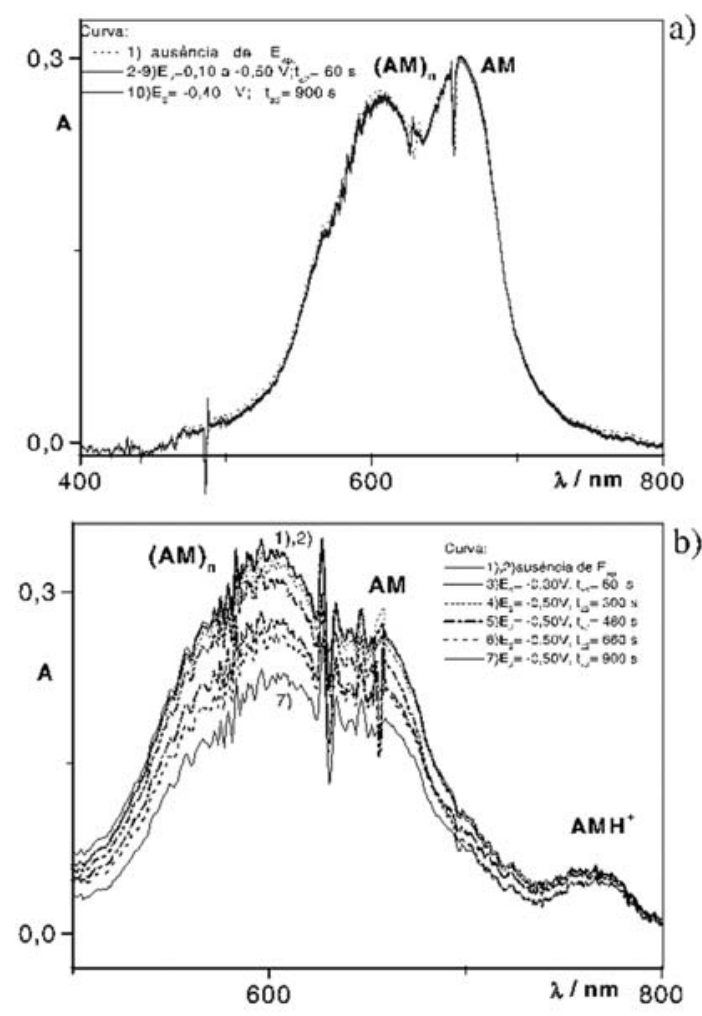

Figura 3. Espectros de absorção na região do visível dos filmes do compósito MM-AM obtidos durante e na ausência da aplicação dos transientes I x t, para diferentes condições experimentais: a) compósito contendo CTACl na região interlamelar; b) ausência do $\mathrm{CTACl}$ na região interlamelar. Condições para obtenção dos transientes descritos na figura. $\mathrm{T}=(25 \pm 1)$ oC.

Na Figura 3b) estão representados os espectros de absorção para o eletrodo de vidro ITO modificado com o filme do compósito MM-AM na ausência do CTACl na região interlamelar. A curva
1 da Figura 3b) foi obtida na ausência de Eap., revelando as espécies absorventes presentes no sistema: enquanto que o espectro 2 (superposto ao 1) foi varrido imediatamente após a remoção do potencial aplicado para a obtenção da curva $7\left(\mathrm{E}_{2}=\right.$ $\left.-0,5 \mathrm{~V}, \mathrm{t}_{\mathrm{a} 2}=900 \mathrm{~s}\right)$. Com a remoção do potencial observou-se o retorno instantâneo do sistema à sua condição inicial, ausência de potencial aplicado; sendo os espectros obtidos totalmente superponíveis, como observado a partir da Figura 3b). A curva 3, por sua vez, foi obtida para tempos curtos, $\mathrm{t}_{\mathrm{a} 2}=60 \mathrm{~s}$ e $\mathrm{E}_{2}=-0,3 \mathrm{~V}$. Este espectro é representativo de um conjunto de espectros superpostos, todos obtidos em condições experimentais idênticas, exceto para os valores de $\mathrm{E}_{2}=0,1,-0,15$ e $-0,20 \mathrm{~V}$. Tal superposição revela a ausência de atenuação do pico de máximo de absorção das espécies ((AM)n em 600 nm; AM em $660 \mathrm{~nm}$ ), para intervalo de tempo de $60 \mathrm{~s}$, situação muito semelhante ao observado a partir dos espectros da Figura 3a).

Os espectros obtidos para transientes de tempo $\mathrm{t}_{\mathrm{a} 2}$, superior a $60 \mathrm{~s}, \mathrm{t}_{\mathrm{a} 2}=300,480,660$ e $900 \mathrm{~s}$, para $E_{1}=0,20 \mathrm{~V}$ e $E_{2}=-0,50 \mathrm{~V}$, curvas $4-7$, em comparação com $t_{\mathrm{a} 2}=60 \mathrm{~s}$ ( curva 3 ), revelam uma contínua atenuação do espectro. A máxima atenuação, observada na intensidade dos picos de absorção das espécies (AM)n em 600 nm e AM em $660 \mathrm{~nm}$, foi de $30 \%$, e foi obtida para o par $\mathrm{t}_{2}, \mathrm{E}_{2}: 900$ s, - $0,50 \mathrm{~V}$. Comportamento desta natureza pode ser comparado aos resultados apresentados na literatura para o estudo eletrocrômico de filmes de AM - nafion e polianilina - AM - nafion [10,12,13]. Para tais sistemas o processo de atenuação requer um intervalo de tempo ( $\leq 180 \mathrm{~s})$ consideravelmente menor do que o exigido para o compósito inorgânico MM-AM (900 s). Entretanto a etapa lenta do processo está na de conversão da forma leuco à forma oxidada do AM.

A atenuação observada para o sistema inorgânico, por sua vez, é da ordem de $30 \%$, e constitui-se num valor ainda pequeno quando comparado a conversão obtida para os sistemas AM - nafion e polianilina - AM - nafion [10,12,13], para os quais a eficiência é de $100 \%$. Porém, o sistema inorgânico apresenta, com a remoção do potencial aplicado, um retorno instantâneo à condição inicial. 


\section{Conclusões}

A adsorsão prévia do monômero não protonado, AM, permite o aparecimento do par redox $\mathrm{AB}$, apenas para $\mathrm{CAM} \geq 16 \mu \mathrm{mol} \mathrm{L}-1$ e para um número elevado de ciclos (50). Este fato sugere a necessidade do corante estar presente nas camadas interiores do filme, processo este que ocorre como função do tempo.

A incorporação do surfactante CTACl permitiu: 1) a obtenção de filmes mecanicamente estáveis; 2) sugerir que o monômero protonado $\left(\mathrm{AMH}^{2+}\right)$, presente nos sítios ácidos da argila esteja participando no processo de transferência de elétrons e, portanto, sendo responsável pela conseqüente definição do perfil dos voltamogramas cíclicos.

O estudo espectroeletroquímico revelou que, considerando a aplicação de transientes para: 1) tempo de amostragem $t_{a 2}=60 \mathrm{~s}$, os eletrodos modificados contendo o CTACl incorporado ou não a região interlamelar (metodologias II-4 e II-3, respectivamente) não apresentam atenuação dos picos das espécies absorventes, revelando a ausência da espécie leuco do AM; 2) tempos significativamente maiores $t_{a 2}=900 \mathrm{~s}$, o eletrodo preparado de modo a não conter o $\mathrm{CTACl}$ na camada interlamelar (presença da espécie $\mathrm{AMH}^{2+}$ ) apresentou uma considerável atenuação do pico de absorção da espécie monoprotonada, e o retorno instantâneo à condição inicial com a remoção do $\mathrm{E}_{\text {ap }}$.

\section{Agradecimentos}

Os autores agradecem à FUNDUNESP e à FAPESP o apoio financeiro.

SOUZA, G. R.; FERTONANI, F. L.; PASTRE, I. A. Espectroelectrochemical Behavior Of Clay-Dye Structured Systems.

\section{Abstract}

The eletrochemical and espectroelectrochemical behavior of the montmorilonita methylene-blue system (MM-AM)/ AMsolution have been investigated using cyclic volta mmetry and transient ( $\mathrm{I} x \mathrm{t})$ techniques on modified ITO glass electrodes by film of composite (MM-AM) mechanically deposited. These films were prepared by three way: 1- in the absence of surfactant CTACl (cetyltrimethyl-ammonuim chloride); 2- with incorporation of CTACl into the interlayer region; and 3-in the presence of CTACl, however, not incorporated into the interlayer region. The spectroelectrochemistry technique was used for characterization and study of the formed films. The experimental results allowed assumptions as follows: 1- films prepared with AM previous incorporated into interlayer region supply a more efficient electrochemistry answer with the appearance of a couple of well defined peaks; 2$\mathrm{CTACl}$, in general, allows to obtain mechanically stable films; 3- when CTACl is previously included into interlayer region it doesn't exist intercalated protonated specie, $\mathrm{AMH}+$; 4- when protonated specie is absent the composite doesn't shows electrochromic behavior.

Keywords: Clays, spectroelectrochemistry, cyclic voltammetry, montmorillonite, methylene blue and modified electrodes.

\section{Referências Bibliográficas}

[1] BERGMANN, K.; OKONSKI, C. T. A spectroscopic study of methylene blue monomer, dimer, and complexes with montmorillonite. J. Phys. Chem., v. 67, p. 2169, 1963.

[2] BREEN, C.; LOUGHLIN, H. The competitive adsorption of methylene-blue on to na-montmorillonite from binary-solution with n-alkylytrimethylammonium surfactants. Clay Miner., v. 29, p. 775-783, 1994.

[3] BUJDAK, J.; KOMADEL, P. Interaction of methylene blue with reduced charge montmorillonite. J. Phys. Chem. B, v. 101, p. 9065-9068, 1997.

[4] CASTROACUNA, C. M.; FAN, F. R. F.; BARD, A. J. Clay modified electrodes .8. Electrochemical-behavior at montmorillonite and polypyrrole montmorillonite films on platinum and glassy-carbon electrodes. J. Electroanal. Chem., v. 234, p. 347-353, 1987.

[5] CELIS R.; HERMOSIN, M. C.; CARRIZOSA, M. J.; CORNEJO, J. Clay-herbicide complexes to retard picloram leaching in soil. Int. J. Environ. Anal. Chem., v. 82, p. 503517,2002 .

[6] CELIS, R.; HERMOSIN, M. C.; CARRIZOSA, M. J.; Ecl. Quím., São Paulo, 28(1): 77-83, 2003 
CORNEJO, J. Inorganic and organic clays as carriers for controlled release of the herbicide hexazinone. J. Agr. Food Chem., v. 50, p. 2324-2330, 2002.

[7] COSNIER, S.; LELOUS, K. A new strategy for the construction of amperometric dehydrogenase electrodes based on laponite gel-methylene blue polymer as the host matrix. J. of Electroanal. Chem., v. 406, p. 243-246, 1996.

[8] CSOBAN, K.; PARKANYI-BERKA, M.; JOO, P.; BEHRA, PH. Sorption experiments of $\mathrm{Cr}$ (III) onto silica. Colloids Surfaces A, v. 141 p. 347-364, 1998.

[9] HU, S. S. Electrocatalytic reduction of molecular oxygen on a sodium montmorillonite-methyl viologen carbon paste chemically modified electrode. J. Electroanal. Chem., v. 463, p. 253-257, 1999.

[10] JOHN, S. A.; GOBI, K. V.; RAMASUBBU, A.; RAMARAJ, R. Photoinduced electron-transfer reactions at methylene-blue adsorbed nafion and clay coated electrodes. Research Chem. Intermediates, v. 18, p. 203-210, 1992.

[11] KAMAT, P. V. Electrochemistry and photoelectrochemistry of dye-incorporated clay-modified electrode. J. Electroanal. Chem., v. 163, p. 389-394, 1984.

[12] KUWABATA, S.; MITSUI, K.; YONEYAMA, H. Preparation of polyaniline films doped with methylene blue-bound nafion and the electrochromic properties of the resulting films. J. Electroanal. Chem., v. 281 p. 97107, 1990 .

[13] KUWABATA, S.; NAKAMURA, J.; YONEYAMA, H. Dimerization kinetics of methylene-blue incorporated in a nafion film. J. Electroanal. Chem., v. 261, p. 363373,1989

[14] LEI, C. H.; ZHANG, Z.; LIU, H.; KONG, J.; DENG, J. Biosensoring of hydrogen peroxide using new methylene blue $\mathrm{N}$ incorporated in a montmorillonite-modified horseradish peroxidase immobilization matrix as an electron shuttle. Anal. Chim. Acta, v. 332, p. 73-81, 1996.

[15] LIU, W. P.; GAN, J.Y.; YATES, S. R. Influence of herbicide structure, clay acidity, and humic acid coating on acetanilide herbicide adsorption on homoionic clays. $J$. Agr. Food Chem., v. 50, p. 4003-4008, 2002.

[16] MARGULIES, L.; ROZEN, H. Adsorption of methyl green on montmorillonite, J. Mol. Struct., v. 141, p. 219226, 1986 .

[17] MARGULIES, L.; ROZEN, H.; COHEN, E. "Energy transfer at the surface of clays and protection of pesticides from photodregadation. Nature, v. 315, p. 658-659, 1985.

[18] NENNEMANN, A.; MISHAEL, Y.; NIR, S.; RUBIN, B.; POLUBESOVA, T.; BERGAYA, F.; VAN DAMME, H.; LAGALY, G. Clay based formulations of metolachlor with reduced leaching. App. Clay Sci., v. 18, p. 265-275, 2001.

Ecl. Quím., São Paulo, 28(1): 77-83, 2003
[19] ONIKUBO, T.; LIN, R. J.; KANEKO, M. In-situ twin-electrode spectrocyclic voltammetry with a cell composed of a pair of nafion(r)-coated indium tin oxide electrodes each incorporating ru(bpy)3(2+) and methylviologen. J. Electroanal. Chem., v. 361, p. 143$148,1993$.

[20] RAUPACH, M.; EMERSON, W. W.; SLADE, P. G. The arrangement of paraquat bound by vermiculite and montmorillonite. J. Colloid Interface Sci., v. 69, p. 398408, 1979.

[21] RYTWO, G.; NIR, S.; MARGULIES, L. Interactions of monovalent organic cations with montmorillonite adsorption studies and model-calculations. Soil Sci. Soc. Am. J., v. 59, p. 554-564, 1995.

[22] SHEN, B. E.; PENG, T. Z.; WANG, H. W. The electrochemical-behavior of cationic and anionic dye-clay modified electrodes. Electrochim. Acta, v. 39, n. 4, p. 527$530,1994$.

[23] SHYU, S. C.; WANG, C. M. Characterizations of ironrich clay modified and their applications in optical recognition. J. Electroanal. Chem., v. 440, p. 27-33, 1997.

[24] SONG, C. J.; VILlEMURE, G. Preparation of claymodified electrodes by electrophoretic deposition of clay films. J. Electroanal. Chem., v. 462, p. 143-149, 1999.

[25] VILlEMURE, G., BARD, A. J. Clay modified electrodes .9. Electrochemical studies of the electroactive fraction of adsorbed species in reduced-charge and preadsorbed clay films. J. Electroanal. Chem., v. 282, p. 107-121, 1990.

[26] WHITE, J. R.; BARD, A. J. Clay modified electrodes .4. The electrochemistry and electron-spin-resonance of methyl viologen incorporated into montmorillonite films. J. Electroanal. Chem., v. 197, p. 233-244, 1986.

[27] ZEN, J. M.; CHEN, P. J. A selective voltammetric method for uric acid and dopamine detection using clay modified electrodes. Anal. Chem., v. 69, p. 5087-5093, 1997.

[28] ZEN, J. M.; KUMAR, A. S.; CHEN, H. W. Electrochemical formation of Prussian blue in natural ironintercaleted clay and cinder matrixes. Electroanalysis, v. 12 , p. 542 - 545, 2000.

Recebido em: 28/02/03 Aceito em: 23/04/03 